\title{
Phenolic acid metabolites as biomarkers for tea- and coffee-derived polyphenol exposure in human subjects
}

\author{
Jonathan M. Hodgson ${ }^{1 *}$, Shin Yee Chan ${ }^{1}$, Ian B. Puddey ${ }^{1}$, Amanda Devine ${ }^{2}$, \\ Naiyana Wattanapenpaiboon ${ }^{3}$, Mark L. Wahlqvist ${ }^{3}$, Widjaja Lukito ${ }^{4}$, Valerie Burke ${ }^{1}$, \\ Natalie C. Ward ${ }^{1}$, Richard L. Prince ${ }^{2}$ and Kevin D. Croft ${ }^{1}$ \\ ${ }^{1}$ University of Western Australia School of Medicine and Pharmacology and the Western Australian Institute for \\ Medical Research at Royal Perth Hospital, Perth, Western Australia, Australia \\ ${ }^{2}$ University of Western Australia School of Medicine and Pharmacology and the Western Australian Institute for \\ Medical Research at QEII Medical Centre, Perth, Western Australia, Australia \\ ${ }^{3}$ The Asia Pacific Health and Nutrition Centre, Monash Asia Institute, Monash University, Clayton, Victoria, \\ Australia \\ ${ }^{4}$ SEAMEO-TROPMED Regional Center for Community Nutrition, University of Indonesia, Jakarta, Indonesia
}

(Received 29 July 2003 - Revised 8 October 2003 - Accepted 10 October 2003)

\begin{abstract}
Tea and coffee are rich in polyphenols with a variety of biological activities. Many of the demonstrated activities are consistent with favourable effects on the risk of chronic diseases. 4-O-methylgallic acid (4OMGA) and isoferulic acid are potential biomarkers of exposure to polyphenols derived from tea and coffee respectively. 4OMGA is derived from gallic acid in tea, and isoferulic acid is derived from chlorogenic acid in coffee. Our major objective was to explore the relationships of tea and coffee intake with $24 \mathrm{~h}$ urinary excretion of 4OMGA and isoferulic acid in human subjects. The relationships of long-term usual (111 participants) and contemporaneously recorded current (344 participants) tea and coffee intake with $24 \mathrm{~h}$ urinary excretion of 4OMGA and isoferulic acid were assessed in two populations. 4OMGA was related to usual $(r 0.50, P<0.001)$ and current $(r 0.57, P<0.001)$ tea intake, and isoferulic acid was related to usual $(r 0 \cdot 26, P=0.008)$ and current $(r 0 \cdot 18, P<0.001)$ coffee intake. Overall, our present results are consistent with the proposal that 4OMGA is a good biomarker for black tea-derived polyphenol exposure, but isoferulic acid may be of limited usefulness as a biomarker for coffee-derived polyphenol exposure.
\end{abstract}

Tea: Coffee: Polyphenols: Phenolic acids: Biomarkers

Tea and coffee are widely consumed beverages. They can be major dietary sources of poly-hydroxylated phenolic compounds (polyphenols), including phenolic acids and flavonoids. Tea contains primarily flavonoids, as well as up to $15 \%(25-50 \mathrm{mg}$ per cup) total polyphenols present as non-esterified or esterified gallic acid (Hodgson et al. 2000a; Shahrzad et al. 2001). Coffee polyphenols are almost entirely chlorogenic acid (100-200 mg per cup; Clifford, 1999). Polyphenols have a range of activities (Morton et al. 2000) that might have a positive impact on human health. Results of population studies showing a higher intake of flavonoids to be associated with lower risk for chronic diseases would support this hypothesis (Hertog et al. 1995; Knekt et al. 2002).

The identification of biomarkers of dietary polyphenol exposure would provide an additional tool to investigate relationships with chronic disease-related endpoints.
Several potential biomarkers of exposure to tea- or coffee-derived polyphenols have been identified (Kivits et al. 1997; Hodgson et al. 2000a; Rechner et al. 2001; Shahrzad et al. 2001; Meng et al. 2002). These include specific $O$-methylated polyphenols derived from in vivo polyphenol metabolism, such as 4- $O$-methylgallic acid (4OMGA) and isoferulic acid (Hodgson et al. 2000a; Rechner et al. 2001; Shahrzad et al. 2001; Meng et al. 2002).

A major metabolite of gallic acid in human subjects is 4OMGA (Shahrzad \& Bitsch, 1998; Hodgson et al. 2000a; Shahrzad et al. 2001). 4OMGA has been used to assess compliance in intervention studies (Hodgson et al. 2000b; Caccetta et al. 2001; Hodgson et al. 2001, $2002 a, b)$, and as a marker of tea-derived polyphenol metabolism (Hodgson et al. 2003). 4OMGA has been suggested as a biomarker of tea intake (Hodgson et al. 
$2000 a$ ), and may be a good marker for tea-derived polyphenol exposure, incorporating intake and absorption. The relationship between 4OMGA and tea intake has not been assessed within populations.

The chlorogenic acid in coffee is present primarily as 5caffeoyl quinic acid (chlorogenic acid), which is an ester of caffeic acid with quinic acid (Herrmann, 1989; Clifford, 1999). Isoferulic acid has been identified as a specific metabolite of dietary caffeic acid derivatives, and was suggested as a useful biomarker for intake and metabolism of caffeic acid derivatives from dietary sources (Rechner et al. 2001). Since coffee is a rich dietary source of chlorogenic acid, we proposed that isoferulic acid might be useful as a biomarker for coffee intake and coffee-derived polyphenol exposure. The relationship between isoferulic acid and coffee intake has not been assessed within populations.

The ideal relationship between any exposure and its marker is linear. Our major objective was to explore relationships of $24 \mathrm{~h}$ urinary excretion of 4OMGA and isoferulic acid with usual and current tea and coffee intake in human subjects.

\section{Methods}

\section{Participants and design}

The relationships of 4OMGA and isoferulic acid with usual (group 1) and contemporaneously recorded current (group 2) tea and coffee intake were investigated within two groups of subjects. For both groups, tea and coffee intake were assessed as number of cups. Almost all tea intake was black tea with added milk and most coffee intake was instant coffee. Tea and coffee intakes were not subcategorized due to small numbers within subcategories. The prevalence of gastrointestinal or renal disease (which may influence phenolic acid metabolism) within the populations was very low $(<2 \%)$ and therefore unlikely to influence observed relationships.

Group 1. Group 1 included 111 participants (fortythree men and sixty-eight women) with mean age 55.8 (95\% CI 53.1, 58.5) years, recruited as a representative sample of Anglo-Celtic men and women residing in Melbourne, Australia (Dalais et al. 1998; Kouris-Blazos et al. 1999). Tea and coffee intake were assessed using a food-frequency questionnaire (Kouris-Blazos et al. 1996). 4OMGA and isoferulic acid were measured in a $24 \mathrm{~h}$ urine sample collected at the time that participants filled in the food-frequency questionnaire. The relationships of usual tea and coffee intake with urinary excretion of 4OMGA and isoferulic acid respectively were assessed.

Group 2. Group 2 included 344 participants (fifty men and 294 women) with mean age 71.7 (95\% CI 70.8, 72.6) years, recruited to two studies. The first of these studies included 275 women with mean age 75.1 (95\% CI 74.8, 75.4) years recruited from the Western Australian general population of women $>70$ years of age (Dick et al. 2002). All participants were healthy and did not have any medical conditions likely to influence 5-year survival rate. Although the participants entering the study were weighted in favour of those in higher socio-economic categories, they did not differ from the whole population in health resource utilization (Bruce et al. 2002). Tea and coffee intake were assessed using a $24 \mathrm{~h}$ diet recall administered by a single interviewer. Informed consent was obtained and the Human Rights Committee of the University of Western Australia approved the study. The second study involved sixty-nine participants (fifty men and nineteen women) with mean age 58.0 (95\% CI 55.3, 60.7) years from the Western Australia general population in a cross-sectional and case-control study. Participants involved in the present study were recruited on the basis of having normal or elevated blood pressure. Current tea and coffee intakes were assessed using a $24 \mathrm{~h}$ beverage intake diary. Informed consent was obtained and the Royal Perth Hospital Ethics Committee approved the study.

For both studies a $24 \mathrm{~h}$ urine sample was collected for the period corresponding to that for which tea and coffee intake were assessed. Relationships observed for tea and coffee intake with 4OMGA and isoferulic acid excretion respectively were almost identical within the two studies. Inclusion of participants from both these studies in group 2 allowed analysis in both men and women.

\section{Measurement of 4-O-methylgallic acid and isoferulic acid}

The 4OMGA and isoferulic acid concentrations were measured in $24 \mathrm{~h}$ urine samples, which had been frozen at $-80^{\circ} \mathrm{C}$ before analysis, using a method described by Hodgson et al. (2000a). Briefly, phenolic acids were extracted from urine samples following enzyme hydrolysis using $\beta$-glucuronidase, which also has sulfatase activity (catalogue no. G7017; Sigma, Castle Hill, NSW, Australia). A large proportion of the phenolic acids are present in urine as glucuronides, and some may be present as sulfates or in the non-esterified form. Therefore, the measurements represent total phenolic acid concentrations. Trimethylsilyl derivatives of phenolic acids were made using $\mathrm{N}, \mathrm{O}$-bis(trimethylsilyl)trifluoroacetamide-pyridine $(50: 50, \mathrm{v} / \mathrm{v})$.

Concentrations of 4OMGA and isoferulic acid were analysed on a Hewlett-Packard HP 6890 GC coupled to an HP 5973 MS (Hewlett-Packard, Palo Alto, CA, USA). An HP-5 MS column $(25 \mathrm{~m} \times 0.20 \mathrm{~mm}, 0.33 \mu \mathrm{m}$ film thickness; Hewlett-Packard) was used with $\mathrm{He}$ as the carrier gas and an inlet pressure of $30 \mathrm{kPa}$. Injections were made in a splitless mode. The initial column temperature of $120^{\circ} \mathrm{C}$ was held for $0.5 \mathrm{~min}$, then increased by $15^{\circ} \mathrm{C} /$ min to $280^{\circ} \mathrm{C}$, where it was held for $5 \mathrm{~min}$. The MS was operated in electron impact $(70 \mathrm{eV})$, and all ions were monitored in the scan mode. Analyte abundances were compared using extracted ion chromatograms.

The identification of 4OMGA and isoferulic acid was based on retention time and mass spectra compared with authentic standards. The authentic standard of 4OMGA was prepared according to previously described procedures (Hodgson et al. 2000a). The authentic standard of isoferulic acid was prepared from isovanillin and malonic acid. Isovanillin $(15 \mathrm{~g}, 0.144 \mathrm{~mol})$ and malonic acid $(30 \mathrm{~g}$, $0.380 \mathrm{~mol})$ were dissolved in pyridine $(30 \mathrm{ml})$ containing piperidine $(0.5 \mathrm{ml})$ and heated under reflux for $3 \mathrm{~h}$. The reaction mixture was poured into excess water containing $5 \mathrm{M}-\mathrm{HCl}$ to remove pyridine, and the white precipitate was filtered and washed with water. The product 
was recrystallized from ethanol (containing acetic acid $(5 \mathrm{ml} / \mathrm{l})$ ) to give white crystals of isoferulic acid (melting point $231^{\circ} \mathrm{C}$; literature melting point $232^{\circ} \mathrm{C}$ ). Product identity was confirmed by MS analysis as the trimethysilyl derivative (major ions m/e $328\left[\mathrm{M}^{+}\right], 323\left[\mathrm{M}^{+}-15\right]$ ).

\section{Statistics}

All statistical analyses were performed using SPSS, version 11.5 (SPSS Inc., Chicago, IL, USA). Results are presented as mean values and $95 \% \mathrm{CI}$ and $P<0.05$ was used as the level of significance. The creatinine-adjusted urinary concentrations of 4OMGA and isoferulic acid were not normally distributed; therefore, log-transformed values were used. Univariate associations were assessed using Spearman's rank correlation. Linear regression was used to investigate the relationships of 4OMGA and isoferulic acid with tea and coffee intake after adjustment for potential confounding factors. Covariates considered in these models included age, gender and source of study participants (group 2).

\section{Results}

The relationships of 4OMGA and isoferulic acid with usual tea and coffee intake were assessed in group 1 , and with current intake in group 2. The tea and coffee intake, and the $24 \mathrm{~h}$ urinary excretion of 4OMGA and isoferulic acid, for each population are presented in Table 1 .

Urinary 4OMGA excretion was significantly related to both usual tea intake $(r 0.50, P<0.001)$ and current tea intake $(r 0 \cdot 57, P<0 \cdot 001)$. Urinary isoferulic acid excretion was significantly related to both usual coffee intake $(r 0 \cdot 26$, $P=0.008)$ and current coffee intake $(r 0.18, P<0.001)$. Adjustment for age, gender and source of study participants (group 2), did not alter the observed relationships.

The relationships of 4OMGA and isoferulic acid with current tea and coffee intake respectively are summarized in Fig. 1. A cut-off concentration for 4OMGA excretion of $25 \mu \mathrm{g} / \mathrm{mmol}$ creatinine had $82 \%$ sensitivity and $81 \%$ specificity for prediction of tea-drinking status. A cut-off

Table 1. Tea and coffee intake, and $24 \mathrm{~h}$ urinary excretion of 4-Omethylgallic acid and isoferulic acid for two groups: one in which usual tea and coffee intake was assessed and one in which current tea and coffee intake was assessed

(Mean values and $95 \%$ confidence intervals)

\begin{tabular}{|c|c|c|c|c|}
\hline & \multicolumn{2}{|c|}{$\begin{array}{l}\text { Group } 1 \text { (n 111): } \\
\text { usual intake }\end{array}$} & \multicolumn{2}{|c|}{$\begin{array}{l}\text { Group } 2 \text { (n 344): } \\
\text { current intake }\end{array}$} \\
\hline & Mean & $95 \% \mathrm{Cl}$ & Mean & $95 \% \mathrm{Cl}$ \\
\hline $\begin{array}{l}\text { Tea intake (no. of } \\
\text { cups per } d \text { ) }\end{array}$ & 2.49 & $2.05,2.93$ & 1.99 & $1 \cdot 80,2 \cdot 18$ \\
\hline $\begin{array}{l}\text { Coffee intake (no. of } \\
\text { cups per d) }\end{array}$ & 1.56 & $1.23,1.90$ & $1 \cdot 28$ & $0.98,1.52$ \\
\hline $\begin{array}{l}\text { 4-O-Methylgallic acid } \\
\text { ( } \mu \mathrm{g} / \mathrm{mmol} \text { creatinine) }\end{array}$ & $42 \cdot 1 \dagger$ & $29 \cdot 0,61 \cdot 2$ & $38.9 \dagger$ & $30.9,49.3$ \\
\hline $\begin{array}{l}\text { Isoferulic acid } \\
(\mu \mathrm{g} / \mathrm{mmol} \text { creatinine })\end{array}$ & $11 \cdot 1 \dagger$ & $8 \cdot 8,14 \cdot 1$ & $7.8 \dagger$ & $6 \cdot 4,9.5$ \\
\hline
\end{tabular}

${ }^{\star}$ For details of subjects and procedures, see p. 302.

† Geometric mean values.
(A)

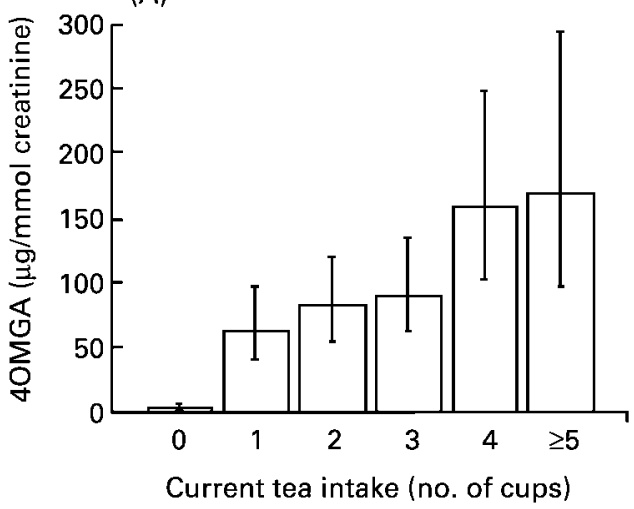

(B)

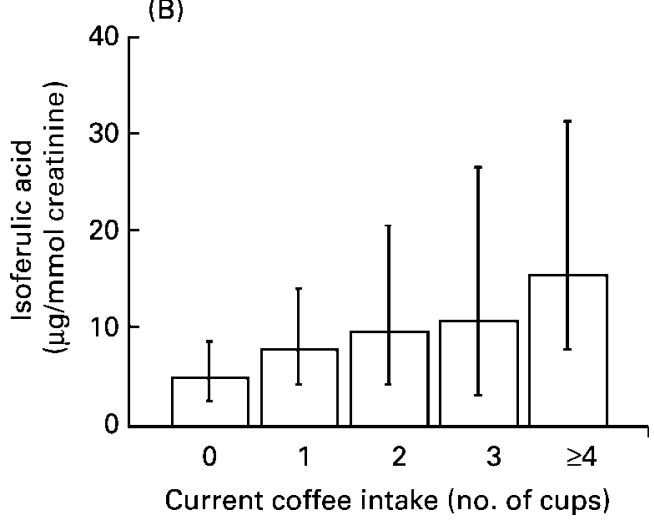

Fig. 1. (A), The effect of the number of cups of current tea intake on excretion of 4-O-methylgallic acid (4OMGA; group 2). (B), The effect of the number of cups of coffee intake on excretion of isoferulic acid (group 2). For details of subjects and procedures, see p. 302. Values are geometric means with $95 \% \mathrm{Cl}$ represented by vertical bars. Linear trends were analysed by linear regression: $(A)$, $P<0.001$; (B), $P=0.001$.

concentration for isoferulic acid excretion of $5 \mu \mathrm{g} / \mathrm{mmol}$ creatinine had $61 \%$ sensitivity and $57 \%$ specificity for prediction of coffee-drinking status.

\section{Discussion}

Tea and coffee are rich dietary sources of polyphenols. All tea derives from the plant Camellia sinensis. Coffee can be obtained from several Coffea species, with Coffea arabica representing most of the world's coffee production. Tea can be processed into a variety of types, although the two main types are black and green. Promoting the enzymatic oxidation of polyphenols produces black teas, whereas enzymes involved in polyphenol oxidation are inactivated in the production of green tea. Production of black tea results in changes to polyphenol structure and composition, including increased non-esterified gallic acid concentrations. Coffee can also be processed and prepared for drinking in a range of ways. Processing and differing methods of preparation of tea or coffee can lead to substantial differences in chemical composition. However, the final beverages contain consistently high concentrations of polyphenols, including gallic acid (tea) and chlorogenic acid (coffee).

Identification of biomarkers of intake of, and exposure to tea- and coffee-derived polyphenols has not been 
straightforward. This has been largely due to the complex nature of many of the polyphenols present in tea, and the lack of knowledge regarding polyphenol metabolism. We proposed that 4OMGA and isoferulic acid might be useful as biomarkers of tea- and coffee-derived polyphenol exposure, and may be used as markers of intake, compliance, polyphenol absorption and metabolism, and to investigate the relationships with disease-related endpoints. 4OMGA and isoferulic acid may have advantages over assessment of intake where an indication of polyphenol intake and absorption or metabolism is required.

Following ingestion of black tea, most urinary 4OMGA is likely to be derived from non-esterified, rather than esterified gallic acid. Much of the gallic acid present in black tea is non-esterified gallic acid, whereas green tea, which has similar total gallic acid present as gallate esters, contains little non-esterified gallic acid (Harbowy \& Ballentine, 1997; Hodgson et al. 2000a). We have previously shown that following a set dose of black and green tea, the increase in urinary 4OMGA was 3-fold greater for black tea (Hodgson et al. 2000b). Non-esterified gallic acid may be absorbed in the small intestine and then transported to the liver, where $O$-methylation and glucuronidation occurs; it is then excreted.

In contrast, most urinary isoferulic acid derives from cleavage of the caffeol quinic acid derivatives. This is likely to occur in the large intestine via esterase activity of gut microflora (Plumb et al. 1999; Rechner et al. 2001). Gallic acid may also be released from larger flavonoids containing gallate esters by the same means. The observed increase in urinary 4OMGA excretion following ingestion of green tea is consistent with this suggestion (Hodgson et al. 2000b). The non-esterified caffeic acid or gallic acid released may then be absorbed, transported to the liver for $O$-methylation to isoferulic acid and 4OMGA respectively; glucuronidation then occurs, followed by excretion. Alternatively, the gut microflora could also be responsible for $O$-methylation, with the compounds absorbed in this form (Chesson et al. 1999). In addition, the phenolic acids may be further metabolized to smaller molecular mass compounds (Scalbert \& Williamson, 2000).

Peak plasma concentrations of phenolic acids occur about 1-2 h after ingestion (Shahrzad et al. 2001; Nardini et al. 2002). The precise time may relate to the location of absorption: small or large intestine. Incorporation into tissues is possible (Youdim et al. 2000), but is likely to be small. The $O$-methylated phenolic acids are then rapidly excreted and fully eliminated from plasma within 12$24 \mathrm{~h}$ (Shahrzad et al. 2001). Therefore, $24 \mathrm{~h}$ urine samples will provide a better indication of short-term consumption pattern and exposure than plasma measurements.

Urinary 4OMGA excretion was related to both usual and current tea intake. As might be expected, urinary 4OMGA predicted more of the variance in current tea intake than usual tea intake. However, the strength of the relationship was only slightly weaker for usual intake, suggesting that current tea consumption strongly predicts usual consumption. Although the mean 4OMGA concentrations were low in participants not drinking tea (Fig. 1(A)), the presence of 4OMGA in a substantial number of these participants is suggestive of other dietary sources. Therefore, some of the unexplained variance may be due to the presence of other dietary sources of gallic acid (Herrmann, 1989; Caccetta et al. 2001), but in comparison with tea, their contribution is likely to be minor. We have previously found that regardless of background diet, urinary excretion of 4OMGA increases at least 3-fold and up to 14-fold following an increase in regular ingestion of black tea by five cups per d (Hodgson et al. 2003). In addition, the 4OMGA response to a given dose of gallic acid is highly variable, there are errors in estimation of intake, and variations in the strength of tea and coffee ingested. However, overall the results are consistent with the proposal that $24 \mathrm{~h}$ excretion of $40 \mathrm{MGA}$ provides a good biomarker of exposure to tea-derived polyphenols, and a reasonable estimate of intake.

Isoferulic acid was significantly related to coffee intake, but the relationship was weak. The results are consistent with the suggestion that isoferulic acid is a specific metabolite derived from chlorogenic acid (Rechner et al. 2001). However, $<7 \%$ of the variance in coffee intake was explained by isoferulic acid excretion, and the absolute increase in isoferulic acid was less than for 4OMGA. This suggests that less of the endproduct of metabolism of chlorogenic acid is in the form of isoferulic acid. The weak relationship may be due to the presence of other dietary sources of caffeic acid derivatives, as well as variability in isoferulic acid excretion in response to a given dose of chlorogenic acid. The presence of isoferulic acid in those not drinking coffee within the population studies is suggestive of other dietary sources. Therefore, isoferulic acid, although a specific metabolite of chlorogenic acid metabolism, appears not to be a major metabolite. Alternatively, it may have undergone further metabolism before urinary excretion. Differences in metabolism between non-esterified and esterified phenolic acids may help to further explain the weak association of isoferulic acid with coffee intake.

Overall, our present results are consistent with the proposal that 4OMGA can be used as a biomarker for tea intake, and may provide a good indication of exposure to tea-derived polyphenols. However, isoferulic acid is quantitatively a minor metabolite in the metabolism of caffeic acid derivatives. Although isoferulic acid was significantly related to coffee intake in the population studies, the relationship was weak, limiting its usefulness as a potential biomarker of intake of coffee-derived polyphenols.

\section{Acknowledgements}

The projects were supported by grants from Healthway, the National Health and Medical Research Council, The Tetley Group Limited and the UWA Research Grants Scheme.

\section{References}

Bruce DG, Devine A \& Prince RL (2002) Recreational physical activity levels in healthy older women: the importance of fear of falling. J Am Geriatr Soc 50, 84-89. 
Caccetta RAA, Burke V, Mori TA, et al. (2001) Red wine polyphenols, in the absence of alcohol, reduce lipid peroxidative stress in smoking subjects. Free Radic Biol Med 30, 636-642.

Chesson A, Provan GJ, Russell WR, et al. (1999) Hydroxycinnamic acids in the digestive tract of livestock and humans. J Sci Food Agric 79, 373-378.

Clifford MN (1999) Chlorogenic acids and other cinnamates nature, occurrence and dietary burden. J Sci Food Agric 79, 362-372.

Dalais FS, Rice GE, Wahlqvist ML, Hsu-Hage BHH \& Wattanapenpaiboon N (1998) Urinary excretion of isoflavonoid phytoestrogens in Chinese and Anglo-Celtic populations in Australia. Nutr Res 18, 1703-1709.

Dick IM, Devine A, Marangou A, et al. (2002) Apolipoprotein E4 is associated with reduced calcaneal quantitative ultrasound measurements and bone mineral density in elderly women. Bone 31, 497-502.

Harbowy ME \& Ballentine DA (1997) Tea chemistry. Crit Rev Plant Sci 16, 415-480.

Herrmann K (1989) Occurrence and content of hydroxycinnamic and hydroxybenzoic acid compounds in foods. Crit Rev Food Sci Nutr 28, 315-347.

Hertog ML, Kromhout D, Aravanis C, et al. (1995) Flavonoid intake and long-term risk of coronary heart disease and cancer in the Seven Countries Study. Arch Int Med 155, $381-386$.

Hodgson JM, Burke V, Beilin LJ, Croft KD \& Puddey IB (2003) Can black tea influence plasma total homocysteine concentrations? Am J Clin Nutr 77, 907-911.

Hodgson JM, Croft KD, Mori TA, et al. (2002a) Regular ingestion of tea does not inhibit in vivo lipid peroxidation in humans. J Nutr 132, 55-58.

Hodgson JM, Morton LW, Puddey IB, Beilin LJ \& Croft KD (2000a) Gallic acid metabolites are markers of black tea intake in humans. J Agric Food Chem 48, 2276-2280.

Hodgson JM, Puddey IB, Burke V, Watts GF \& Beilin LJ (2002b) Regular ingestion of black tea improves brachial artery vasodilator function. Clin Sci 102, 195-201.

Hodgson JM, Puddey IB, Croft KD, et al. (2000b) Acute effects of ingestion of black and green tea on lipoprotein oxidation. Am J Clin Nutr 71, 1103-1107.

Hodgson JM, Puddey IB, Mori TA, et al. (2001) Effects of regular ingestion of black tea on haemostasis and cell adhesion molecules in humans. Eur J Clin Nutr 55, 881-886.
Kivits GAA, Vandersman FJP \& Tijburg LBM (1997) Analysis of catechins from green and black tea in humans: a specific and sensitive colorimetric assay of total catechins in biological fluids. Int J Food Sci Nutr 48, 387-392.

Knekt P, Kumpulainen J, Jarvinen R, et al. (2002) Flavonoid intake and risk of chronic diseases. Am J Clin Nutr 76, $560-568$.

Kouris-Blazos A, Gnardellis C, Wahlqvist ML, et al. (1999) Are the advantages of the Mediterranean diet transferable to other populations? A cohort study in Melbourne, Australia. $\mathrm{Br} \mathrm{J}$ Nutr 82, 57-61.

Kouris-Blazos A, Wahlqvist ML, Trichopoulou A, Polychronopoulos E \& Trichopoulos D (1996) Health and nutritional status of elderly Greek migrants to Melbourne, Australia. Age Ageing 25, 177-189.

Meng XF, Sang SM, Zhu NQ, et al. (2002) Identification and characterization of methylated and ring-fission metabolites of tea catechins formed in humans, mice, and rats. Chem Res Toxicol 15, 1042-1050.

Morton LW, Abu-Amsha CR, Puddey IB \& Croft KD (2000) Chemistry and biological effects of dietary phenolic compounds: relevance to cardiovascular disease. Clin Exp Pharmacol Physiol 27, 152-159.

Nardini M, Cirillo E, Natella F \& Scaccini C (2002) Absorption of phenolic acids after coffee consumption. J Agric Food Chem 50, 5735-5741.

Plumb GW, Garcia-Conesa MT, Kroon PA, et al. (1999) Metabolism of chlorogenic acid by human plasma, liver, intestine and gut microflora. J Sci Food Agric 79, 390-392.

Rechner AR, Spencer JP, Kuhnle G, Hahn U \& Rice-Evans CA (2001) Novel biomarkers of the metabolism of caffeic acid derivatives in vivo. Free Radic Biol Med 30, 1213-1222.

Scalbert A \& Williamson G (2000) Dietary intake and bioavailability of polyphenols. J Nutr 130, 2073S-2085S.

Shahrzad S, Aoyagi K, Winter A, Koyama A \& Bitsch I (2001) Pharmacokinetics of gallic acid and its relative bioavailability from tea in healthy humans. J Nutr 131, 1207-1210.

Shahrzad S \& Bitsch I (1998) Determination of gallic acid and its metabolites in human plasma and urine by high-performance liquid chromatography. J Chromatogr B 705, 87-95.

Youdim KA, Martin A \& Joseph JA (2000) Incorporation of the elderberry anthocyanins by endothelial cells increases protection against oxidative stress. Free Radic Biol Med 29, $51-60$. 